\title{
How we recall (or don't): the hippocampal memory machine and anesthetic amnesia
}

\section{Comment nous nous souvenons (ou ne nous souvenons pas): la machine à mémoire hippocampique et l'amnésie anesthésique}

\author{
Misha Perouansky, MD • Robert A. Pearce, MD
}

Received: 2 July 2010/Accepted: 26 October 2010/Published online: 18 December 2010

(c) Canadian Anesthesiologists' Society 2010

\begin{abstract}
Purpose The hippocampal formation occupies a central position for the processing of sensory input into learned, remembered, and consciously retrievable information. The mechanisms by which anesthetic drugs interfere with these processes are now emerging. We review the current understanding of the role of the hippocampal formation in the generation of memory traces and how anesthetics might interfere with its function.

Clinical features Intraoperative amnesia is a desired endpoint of general anesthesia from the perspective of both the patient and the practitioner. "Intraoperative awareness with recall" can result when learning and memory do occur. In addition, anesthetics are capable of inducing a state of "conscious amnesia" that can provide insight into the workings of the brain and might be useful clinically.

Conclusions Anesthesiologists routinely induce the most fascinating pharmacologic effects in existence, the reversible interference of anesthetics with higher cognitive functions. Understanding how the drugs in our custody exert their effects should be our contribution to mankind's universal knowledge base.
\end{abstract}

\section{Résumé \\ Objectif La formation hippocampique joue un rôle central dans le traitement et la transformation des informations sensorielles en informations apprises,}

This article is accompanied by an editorial. Please see Can J Anesth 2011; 58(2).

M. Perouansky, MD $(\bowtie) \cdot$ R. A. Pearce, MD Department of Anesthesiology, University of Wisconsin School of Medicine and Public Health, B6/319 Clinical Science Center, 600 Highland Avenue, Madison, WI 53792-3272, USA

e-mail: mperouansky@wisc.edu remémorées et consciemment récupérables. Aujourd'hui, les mécanismes par lesquels les anesthésiques interfèrent avec ces processus deviennent plus connus. Dans cet article, nous passons en revue notre compréhension actuelle $d u$ rôle de la formation hippocampique dans la génération d'engrammes et de la façon dont les agents anesthésiques pourraient interférer avec son fonctionnement.

Éléments cliniques L'amnésie peropératoire est un résultat souhaité de l'anesthésie générale du point de vue du patient aussi bien que de celui du médecin. La « conscience peropératoire avec souvenir " peut survenir lorsqu'il y a apprentissage et mémoire. En outre, les anesthésiques peuvent induire un état «d'amnésie consciente» qui pourrait procurer un aperçu du fonctionnement du cerveau et avoir une utilité clinique.

Conclusion Les anesthésiologistes induisent de façon routinière les effets pharmacologiques les plus fascinants qui soient, soit l'interférence réversible des anesthésiques avec les fonctions cognitives supérieures. Comprendre la façon dont les médicaments sous notre responsabilité exercent leurs effets devrait constituer notre contribution à la base de connaissances universelle de l'humanité.

In the course of the 20th century, as mankind was developing advanced tools to scientifically investigate higher cognitive function, the hippocampal formation (HF) advanced from a lowly olfactory structure to the seat of a cognitive map forming the cornerstone of orientation in space and time. The HF, which encompasses the hippocampus proper (CA1, CA2, and CA3 regions), as well as the dentate gyrus (DG), the subiculum, and the entorhinal cortex, is now at the centre of the quest to understand the 
brain's ability to learn, remember, and predict. The aim of this paper is to explore how anesthetic drug action in the HF contributes to a cardinal endpoint of anesthesia: intraoperative amnesia.

Drugs used perioperatively can cause amnesia via actions on different molecular targets, only a few of which can be matched to individual drugs with a high degree of certainty. Subpopulations of gamma-aminobutyric acid $(\mathrm{GABA})_{\mathrm{A}}$ receptors containing specific subunits mediate the amnestic effect of benzodiazepines, etomidate, and propofol. Muscarinic receptors are the target of scopolamine. The receptors mediating the amnestic effects of inhalational agents and ketamine are known with less certainty, but they probably include $\mathrm{GABA}_{\mathrm{A}}$ receptors, tandem pore domain potassium channels, and N-methyl-Daspartate (NMDA) type glutamate receptors and synapses. It is unknown, however, how important each of these receptor/transmitter systems is for the endpoint of amnesia. Remarkably, all anesthetics that have been studied induce amnesia at a concentration well below that which induces loss of consciousness, especially for types of memory that require processing by the HF (see below). In practice, this means that a state of "conscious amnesia" can be achieved purposefully. Moreover, amnesia has been shown to be a separate effect from sedation for some intravenous anesthetics (midazolam and propofol) ${ }^{1}$ but not for others (dexmedetomidine). ${ }^{2}$

Together with the olfactory cortex, the HF forms a large part of the allo- or heterotypic cortex, which is morphologically distinguished by having fewer cell layers than the neocortex. In the hippocampus this paucity of layers is most striking, i.e., a single dense pyramidal cell (PC) layer with strictly laminated extrinsic and intrinsic afferent nerve fibres that run parallel to the pial surface and orthogonally to the PCs' apical dendrites (see Fig. 1) forming predominantly unidirectional connections between a series of cortical regions. This morphologically elegant simplicity and the potential to yield neural tissue that can survive $e x$ vivo (as acute slices, isolated neurons, or organotypic tissue cultures) propelled the hippocampus into the forefront of experimental neuroscience - both as a model and as a target in its own right - just when a series of remarkable clinical observations were transforming the study of learning and memory.

\section{The hippocampal memory machine}

From obscurity to limelight

Few transformations in science are so tightly intertwined with the fate of one individual as that of our understanding of the role of the HF in memory. Having lost large parts of his HF to an attempt to surgically cure his intractable epilepsy at the age of 27, the late Henry Gustav Molaison (1926-2008) became known to the world of neuroscience as H.M. - the "unforgettable amnesiac". "His case revolutionized the study of memory. Prior to this time, an influential article by A. Brodal ${ }^{3}$ had exposed the flaws of considering the $\mathrm{HF}$ as part of the rhinenecephalon (the smell brain). An alternative hypothesis, current at that time, placed the HF into a phylogenetically ancient limbic system subserving primarily emotional functions. ${ }^{4}$ The fundamental shift in understanding of the role of the HF was triggered by Scoville \& Milner's description of H.M.'s (and eight other surgical patients') clinical syndrome of "loss of recent memory after bilateral hippocampal lesions". Dr. William B. Scoville was the operating neurosurgeon, Dr. Brenda Milner a clinical neuropsychologist with a $\mathrm{PhD}$ degree completed under the tutelage of Donald Hebb. This publication has received approximately 3,000 citations and is easily accessible, as it was reprinted in $2000 .^{5}$

\section{Hippocampus and episodic memory}

Memory is not a unitary phenomenon (Fig. 2). Even taking into account the distinctions between long-term, working, and short-term; between semantic and declarative; and between explicit and implicit memory, no single easily identifiable type of memory is uniformly dependent on a single delimited brain area - including the hippocampus and the medial temporal lobe. Moreover, the dependence of memory on specific brain structures, especially the hippocampus, varies with the passage of time and with the nature of the learning itself. Learning and memory are of such cardinal importance that the advanced vertebrate brain has evolved alternative back-up strategies for many types of learning that can be co-opted if the preferred pathway is impaired, thereby improving individual fitness and complicating the interpretation of experiments (e.g., see ${ }^{6}$ ). Nevertheless, experiments have been designed to test for the involvement (or lack thereof) of specific structures in different types of learning, resulting in the delineation of the following memory systems (involved anatomical structure): procedural, e.g., motor skills and habits (neostriatum and cerebellum), ${ }^{7}$ emotional (amygdala), ${ }^{8}$ and declarative (HF). In broad terms, declarative memory is described as the capacity for conscious recollection of episodic events and semantic content and, importantly, flexible memory expression (as opposed to habitual). It is also the type of memory system that is most sensitive to anesthetic drugs. The importance of declarative memory for the practitioner is readily apparent.

\footnotetext{
A New York Times, Dec. 5th 2008.
} 


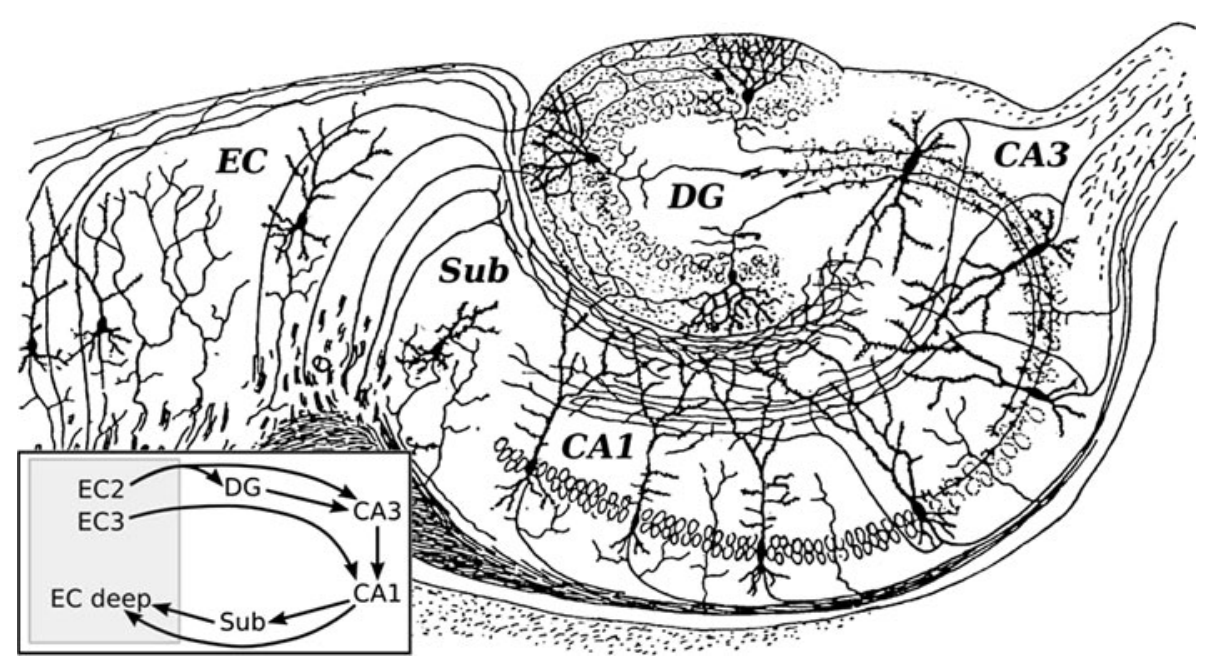

Fig. 1 Schematic representation of the hippocampal tri-synaptic excitatory circuit based on the classic drawing by Santiago Ramon y Cajal. The entorhinal cortex $(E C)$ projects to the hippocampal $C A 1$ area monosynaptically by way of the perforant path and via synapses in the dentate gyrus $(D G)$ and the $C A 3$. All excitatory synapses in this circuit can undergo plastic changes, but the molecular mechanisms differ. The drawing does not capture the intricate inhibitory circuitry that controls excitatory synaptic transmission. (The drawing is downloaded from the public domain by way of the Wikipedia. Originally the drawing was published in 'Histologie du Systeme Nerveux de l'Homme et des Vertebres')
Fig. 2 Schematic overview over the different memory systems. The long-term, declarative, and episodic memory axis is thought to be critically dependent on intact function of the hippocampus/the medial temporal lobe

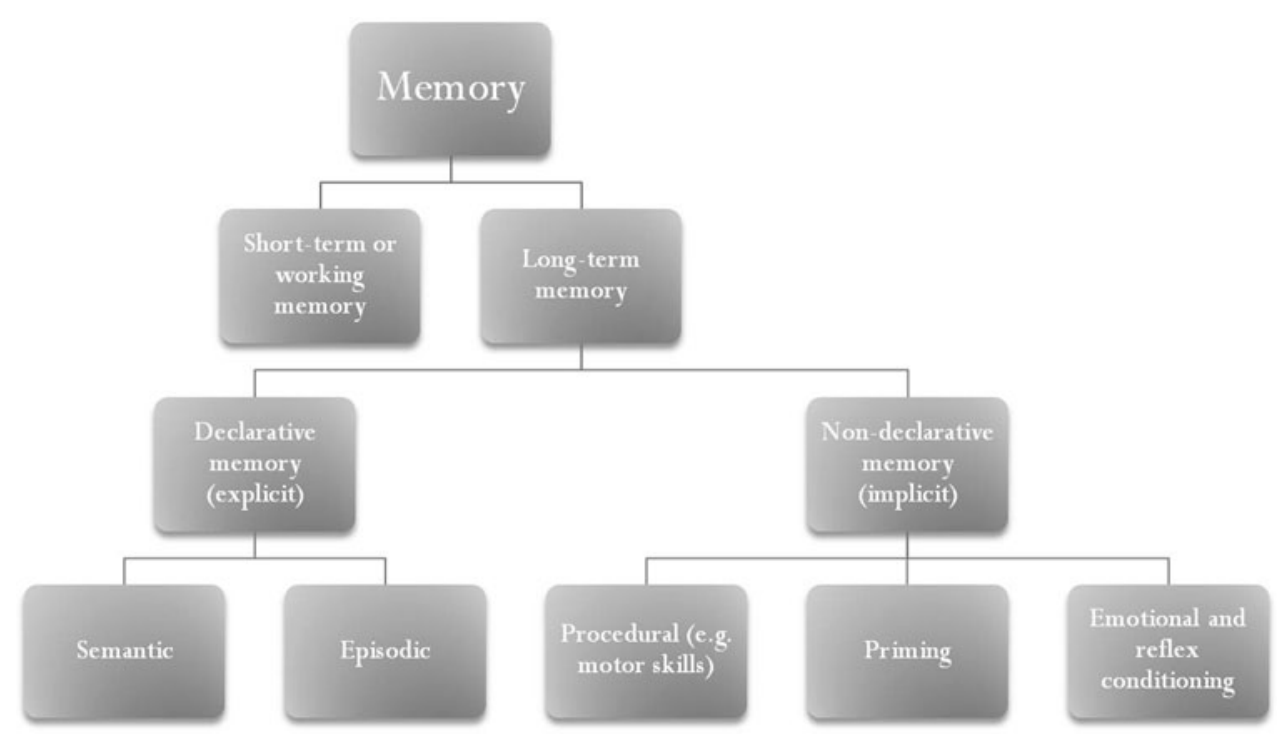

The results from many years of work in a number of leading laboratories have led to the formulation of two theories of fundamental functions of the HF. On the one hand, the HF enables navigation through space by forming cognitive maps of the environment that are stored as place cells and place fields that can be quickly updated if the environment changes. On the other hand, the HF is a key structure for the flexible expression of declarative episodic memories - the term episodic-like is used frequently when applied to animals. ${ }^{9}$ Spatial and episodic memories share the need to organize individual representations in temporal sequences. Without that property, individual locations could not be organized into a map, and memories would lack the what - where - when content characteristic of episodic memories (e.g., I was in the operating room unable to move and undergoing surgery when Doctor $X$ said ...). As a result of these memory functions, the HF is also essential for projecting our experience forward in time/space and thereby predicting the future. Although this discussion focuses on the $\mathrm{HF}$ and its role in learning and memory, it is important to acknowledge that the HF never functions in isolation from the rest of the brain.

Animal models of episodic-like memory

While not undisputed, it is our opinion that animals provide valid experimental systems of episodic memory. These 
models are not necessarily identical to human conscious memory, but they allow the degree of invasiveness necessary for a thorough understanding of anesthetic-mediated suppression of episodic memory trace formation, which is the core of intraoperative awareness or recall. Fear conditioning (FC) in various permutations is a model used widely to investigate learning and memory. ${ }^{10-13}$

Fear conditioning-to-tone and FC-to-context engage largely overlapping sets of brain structures, with the critical difference that the hippocampus is engaged only during FC-to-context. Building on this distinction, Dr. Eger's group at the University of California San Francisco in collaboration with Dr. Fanselow at the University of California Los Angeles determined the dose-response relationship of anesthetics for inhibition of FC in rats, i.e., the median effective concentration required to induce a $50 \%$ effect $\left(\mathrm{EC}_{50 \text {,amnesia }}\right)$, being the memory analogue to minimum alveolar concentration for surgical immobility (MAC). They discovered that the $\mathrm{EC}_{50, \text { amnesia }}$ for contextual $\mathrm{FC}$ was approximately one-half the $\mathrm{EC}_{50}$ for $\mathrm{FC}$ to tone and that both were inhibited at concentrations below the $\mathrm{EC}_{50}$ for loss of righting reflex (the rodent analogue of loss of consciousness). ${ }^{14-16}$ These findings are consistent with results from a variety of other tasks (eyeblink conditioning, inhibitory avoidance, Morris water maze) that indicate that learning tasks are sensitive to disruption by anesthetics at low subhypnotic concentrations, particularly if they require hippocampal processing, ${ }^{17,18}$ and they suggest that some aspect of hippocampal function renders episodic-like memories particularly vulnerable to anesthetic disruption.

Does it follow from these arguments that anesthetics must then act in the hippocampus itself in order to impair episodic memory formation? Not necessarily. Targeted hippocampus-selective manipulations do impair learning and memory. However, actions outside the hippocampus can also affect its proper function, for example, by disturbing the septal $\theta$-rhythm generation and thereby impairing hippocampus-dependent learning and memory.

\section{The hardware}

Important molecular switches

Like in other parts of the mammalian central nervous system (CNS), the neurotransmitters, L-glutamate and GABA, together with a variety of their ionotropic and metabotropic receptors form the backbone of synaptic excitation and inhibition in the hippocampus. These synapses are recognized targets of most general anesthetic drugs and are thought to be mediators of the various components of anesthetic action. The involved receptors for the neurotransmitters display a perplexing heterogeneity, because each receptor is composed of multiple subunits, and multiple isoforms for each subunit exist. However, some receptor/subunit combinations have attracted the attention of scientists studying anesthetic mechanisms because some (in the case of etomidate, all) of the following conditions are present: 1) their function is affected by anesthetics in the relevant concentration range; 2) their specific distribution in the CNS makes it likely that they are important for learning and memory; 3) genetically engineered mice that allow the study of their role have been successfully created.

Among the $\mathrm{GABA}_{\mathrm{A}}$ receptors, those containing the $\alpha 1$ and $\alpha 5$ subunits have been linked most closely with pharmacologically-induced amnesia. The evidence includes both genetic and subunit-specific pharmacological tools. The memory-impairing effect of diazepam in the stepthrough passive avoidance paradigm was reduced in mice carrying a benzodiazepine-sensitive mutation in the $\alpha 1$ subunit. ${ }^{19}$ Similarly, the ability of etomidate to disrupt hippocampal-dependent learning (contextual conditioning and Morris water maze performance) was eliminated in mice lacking the $\mathrm{GABA}_{\mathrm{A}} \mathrm{R} \alpha 5$ subunit. ${ }^{20}$ Whereas, the $\alpha 1$ subunit is the most abundant type of $\mathrm{GABA}_{\mathrm{A}} \mathrm{R} \alpha$ subunit and is distributed throughout the brain, the $\alpha 5$ subunit is expressed most heavily in the hippocampus. This finding provides perhaps the strongest evidence to date that anesthetic action, or at least etomidate action, within the hippocampus itself is critical to anesthetic-induced amnesia.

Synaptic excitation, especially the form mediated by NMDA receptors, is another target of anesthetic action that may contribute to amnesia, at least for some drugs, such as ketamine $^{21}$ and certain inhaled anesthetics. Since NMDA receptor activation depends on both the presence of the endogenous agonist glutamate as well as membrane depolarization provided by preceding or concurrent synaptic excitation, it acts as a "coincidence detector" for concurrent pre- and post-synaptic activation. ${ }^{22}$ Theoretical studies indicate that this is an important characteristic of many types of "memory machines". These receptors and synapses thus form a crucial link in the chain of plasticity that leads to learning and memory. ${ }^{23}$ Anesthetics can interfere with NMDA receptor activation in a number of ways, including inhibition of postsynaptic receptors or interference with presynaptic transmitter release, or indirectly by preventing the level of depolarization required for receptor activation, for example, by enhancing dendritic $\mathrm{GABA}_{\mathrm{A}}$ receptor activation. ${ }^{24}$

It should be mentioned that other receptors, subunit combinations, and/or channels are higher on the agenda for other endpoints of anesthesia - including, for example, the receptor for glycine (immobility) ${ }^{25}$ and the family of tandem pore domain potassium channels (hypnosis, immobility). ${ }^{26,27}$ 
Synaptic plasticity: learning and memory in a dish

An important property of glutamatergic synapses is their ability to undergo use-dependent changes in synaptic strength, which is commonly referred to as plasticity. The persistent strengthening of excitatory synapses following a brief train of stimuli, a phenomenon termed "long-term potentiation" (LTP), was discovered in the hippocampus, and here it has been most thoroughly studied. However, it has been observed at virtually every examined excitatory synapse in the CNS that uses glutamate as a transmitter. A related phenomenon termed "long-term depression" (LTD) is also observed at many synapses, typically elicited by slower longer-lasting trains of electrical stimuli. Synaptic plasticity is widely considered to be the molecular correlate of learning, memory, and related homeostatic processes in the brain.

A wide variety of stimulus protocols can be used to induce LTP. Some, such as the "tetanic stimulation" protocol, which typically involves a one-second train at 100 $\mathrm{Hz}$, are clearly non-physiological. Nevertheless, even these non-physiological models have produced many important insights into the molecular events underlying alterations in synaptic structure and function. Physiologically inspired models, such as the "theta burst" or "theta-associated" stimulation protocols, have been designed to match more closely in vivo firing patterns. Perhaps tellingly, these protocols can produce synaptic potentiation with fewer individual stimuli. It is subject to debate as to which methods most closely approximate processes occurring naturally, i.e., the method that best represents learning and memory is subject to debate.

In the hippocampus, all three excitatory synapses linking the cellular elements of the unidirectional trisynaptic circuit between principal cells of the hippocampus (Fig. 1) can undergo LTP, but the precise molecular expression of plastic changes differs. Glutamatergic synapses on $\mathrm{GABA}_{\text {ergic }}$ (i.e., inhibitory) interneurons can also undergo plastic changes. While synaptic plasticity is expressed at excitatory synapses, it is controlled by inhibitory (e.g., $\mathrm{GABA}_{\text {ergic }}$ ) systems.

Long-term potentiation is a popular (and attractive) model for understanding basic elements of anestheticinduced amnesia. Generally speaking, conditions that reduce inhibition or increase excitation will facilitate LTP. Among many other effects, the potent inhaled anesthetics enhance the $\mathrm{GABA}_{\mathrm{A} \text {-ergic }}$ inhibitory systems and suppress excitatory synaptic transmission. It is unknown as to whether, how, and to what degree these effects contribute to anesthetic amnesia. The effects of inhaled anesthetics on LTP have been studied in vivo and in vitro with conflicting results; in vitro ${ }^{28}$ but not in vivo ${ }^{29}$ LTP can be suppressed with inhaled agents. Reconciling these findings and developing valid models to study the molecular-cellularcircuit mechanisms of drug interference with plasticity and learning and memory are goals of current research in anesthetic mechanisms.

Integrated circuits

Some of the features of the hippocampal wiring that are thought to be distinctive for its function as a memory machine are:

1. Projection neurons from the entorhinal cortex contact the dendrites of hippocampal CA1 PCs via both direct monosynaptic (entorhinal cortex $[\mathrm{EC}]$ to $\mathrm{CA} 1$ PC dendrite, the perforant path) and indirect $(\mathrm{EC} \rightarrow$ $\mathrm{DG} \rightarrow \mathrm{CA} 3 \rightarrow \mathrm{CA} 1$ via the mossy fibres and the Schaffer collaterals). This means that an action potential fired by a neuron in the EC will reach the CA1 neuron twice, but one of the signals will have been delayed and processed in the DG and the CA3 region. This has important functional consequences (see below). Some anatomists argue that the perforant path is such a distinguishing anatomic characteristic that without a topologically identical fibre system there can be no hippocampus-like structure. $^{30,31}$

2. The CA3 PCs excite CA1 PCs and also excite each other via recurrent excitatory connections. This autoassociative network is thought to enable associative representations and pattern completion, elemental processes underlying declarative memory. For a typical CA3 PC, the number of recurrent synapses far exceeds that of all other excitatory inputs.

3. The target of this duplicate excitatory input (the CA1 PCs) sends excitatory projections to the EC both directly and via the subiculum, closing the re-entrant (computational!) loop with the cortex.

4. Multiple other connections to the hippocampus exist. Those from the diencephalon (septum, mammillary bodies, and diagonal band of Broca) are critical for synchronizing the hippocampal network.

Sequential, recurrent, and re-entrant excitatory connections have the propensity to create runaway excitation. However, through tight control by intricate multifaceted inhibitory networks, excitation can be harnessed into useful computation. Since these powerful excitatory loops are notorious for becoming unbalanced, the medial temporal lobe has become the preferred substrate for in vitro studies of epilepsy and the target of surgical attempts to cure this disease. In the healthy brain, a complex array of inhibitory loops consisting of diverse specialized interneurons precisely controls the balance and timing of excitation and inhibition. 


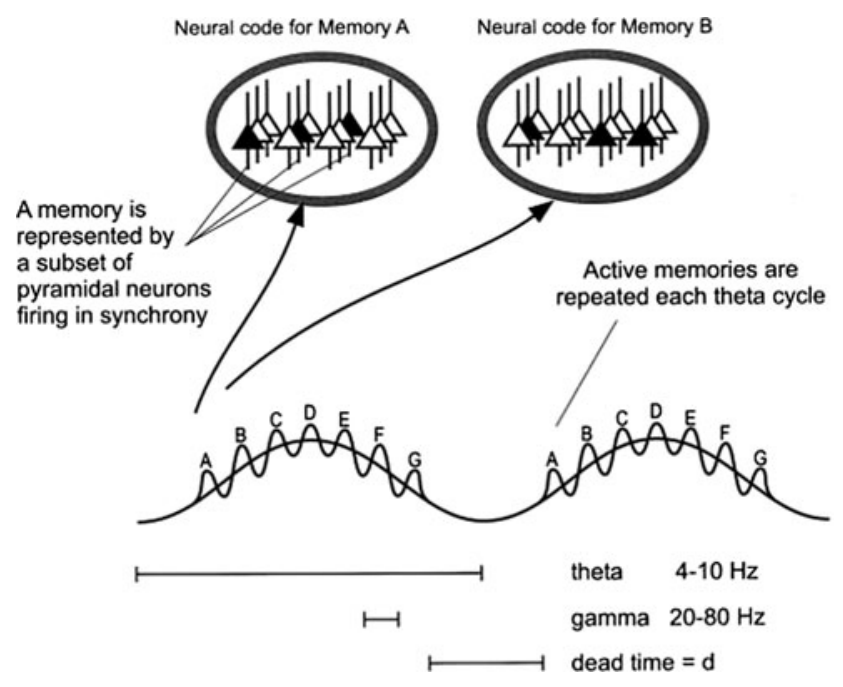

Fig. 3 A model for $\theta$ - $\gamma$-nesting as a means to maintaining items in the working memory buffer. Seven memories $(A-G)$ are multiplexed. Memory $A$, represented by a certain spatial pattern of cell firing, i.e., a cell assembly (oval insert), is active in the first $\gamma$-subcycle of a $\theta$ oscillation, followed by memory $B$ in the next $\gamma$-cycle, etc. After a dead time (d), the seven memories repeat in the subsequent $\theta$-cycle. Reproduced with permission from reference ${ }^{58}$

Software I: cell assemblies

The fundamental insight that the brain's ability to generate a coherent thought derives from the spatiotemporal orchestration of its neurons, i.e., the cell assembly hypothesis, can be traced back to D. Hebb (1949) and even I.P. Pavlov (1927). ${ }^{\text {B }}$ Its centrepiece is the dynamic formation and dissolution of groups of neurons that influence each other's behaviour via the rules of synaptic plasticity, "neurons that fire together wire together". In many cases, reverberating loops allow firing to be more tightly coupled than predicted by the properties of the triggering stimulus acting on a desynchronized system, and to remain active far longer than the triggering input itself (Fig. 3).

Software II: oscillations

The neuronal circuits of the HF interacting with the medial septum and multiple other structures give rise to prominent synchronized neuronal activity patterns that generate extracellular field potentials that coalesce into the electroencephalographic signature of the hippocampus. The bestcharacterized rhythms are the $\theta$-rhythm $(4-10 \mathrm{~Hz})$, which is strongly associated with exploratory activity (typically locomotion) and rapid eye movement (REM) sleep, and the $\gamma$-rhythms (low $\gamma=30-70 \mathrm{~Hz}$; high $\gamma=80-140 \mathrm{~Hz}$ ), which are thought to "bind" together the elements of a mental

\footnotetext{
${ }^{\mathrm{B}}$ Reviewed in 'Rhythms of the Brain' p. 159 by Gyorgi Buzsaki, Oxford Universtity Press 2006.
}

representation into a unitary percept. Other prominent but less periodic patterns are associated with immobility, consummatory behaviour, and slow-wave sleep, including large-amplitude irregular activity (LIA) and small-amplitude irregular activity (SIA). The LIA and SIA, which might be considered "idling" rhythms, are interrupted regularly by a brief (40-120 msec) highly synchronous discharge of CA3 PCs, the sharp wave (SW). Coincident with the SW, 180-220 Hz "ripples" occur in the CA1 region, reflecting rapid firing of CA1 interneurons and synchronous discharge of pyramidal neurons. ${ }^{32}$ Together they are collectively referred to as sharp wave-ripple complexes (SW-R). Similar highly synchronous and brief discharges can be recorded from the DG and are referred to as dentate spikes.

\section{The program: remembering the past, imagining the future}

Different theories exist as to the evolutionary origin of declarative memory. G. Buzsaki proposes that the two components of declarative memory, episodic and semantic, evolved from computational solutions for the demands of navigation on a linear track and in a two-dimensional space, respectively. H. Eichenbaum argues that a multimodal relational memory system, which also proves useful for navigation, was at the origin. Be that as it may, the result in the modern mammalian brain is a flexible memory system capable of rapid storage and retrieval of information that exhibits associativity and can be used inferentially in novel situations, and whose episodic (i.e., sequential) representations of time and space can be woven into multimodal semantic memories that, with the passage of time, become independent of the hippocampus.

The hippocampus implements the declarative memory program in three subroutines that can be allocated to its three macroscopic anatomical components. The internal clocks binding the system together and serving as a reference mechanism for internal (and external) synchronization are the $\theta$-rhythm, which is highly coherent throughout the medial temporal lobe, and the $\gamma$-rhythms, which synchronize firing in the entorhinal cortex and CA1 (high $\gamma$ ) or CA3 and CA1 (low $\gamma$ ), as the task demands dictate. ${ }^{33,34}$

Subroutine 1 - granule cells of the dentate gyrus (DGGCs)

In accordance with theoretical predictions, selective lesions of the DG-GCs or genetic-based impairment of LTP in DG-GCs in experimental animals does not affect overall spatial learning but rather impairs the ability to differentiate between similar-appearing contexts. ${ }^{35,36}$ This process, 
commonly referred to as disambiguation or pattern separation, is thought to be accomplished through a transformation of perforant path input from the EC into a sparse orthogonal representation in which minor differences are amplified and information can be efficiently stored and recalled by the autoassociative circuitry in the CA3 region. The theoretical predictions are in agreement with experimental results showing NMDA receptordependent pattern separation in DG and CA3. ${ }^{35,37}$

Subroutine 2 - CA3: pattern completion, autoassociation

The prominent recursive wiring of the $\mathrm{CA} 3$ region enables not only storage of episodic patterns but also retrieval of a whole representation based on only a fragment of the original. The current thinking is that, due to the extensive recurrent innervations, the bilateral $\mathrm{CA} 3$ areas function as a single computational space that can allow associations between inputs (i.e., information) originating from parts of the neocortex that are widely separated both physically and functionally.

\section{Subroutine 3 - CA1: mismatch detection}

The duplicate excitatory input to CA1 PCs via perforant path and Schaffer collaterals supports a task that is essential for survival, i.e., detection and reaction to novelty in the environment. By comparing stored representations (retrieved in CA3 based on partial cues) with current sensory input from the EC, the CA1 region functions as an associative match-mismatch processor. ${ }^{38,39}$ This processing is implemented by providing differential responses of a given CA1 PC to excitation from the $\mathrm{EC}$ alone $v s$ excitation from EC and CA3. The sequential CA3-EC input effectively leads to action potential firing if it occurs within 40$60 \mathrm{msec}$, i.e., within less than half a $\theta$-cycle. ${ }^{40}$

\section{The HF online: keeping time with the $\theta$-clock}

The presence of $\theta$-rhythm signals that the hippocampus is on-line. This is typically the case whenever the experimental animal is actively exploring its environment, constructing a cognitive map by registering multimodal cues, and forming associations between them. The rhythmic, almost sinusoidal, fluctuation in the extracellular field potential reflects the rhythmic nature of the afferent inputs from the entorhinal cortex and septum, which entrain a circuit that has a propensity to oscillate at this frequency.

Two types of $\theta$-rhythm with different behavioural correlates have been identified based on pharmacologic, physiologic, and genetic means. They are thought to reflect the presence of at least two $\theta$-generators. ${ }^{41}$ "Type 1 ", urethane-sensitive $\theta$-rhythm, occurs during exploratory behaviour and is associated with strong input from the entorhinal cortex via the perforant path connection to the distal dendrites. "Type 2", atropine-sensitive $\theta$-rhythm occurs during alert immobility and is associated with strong input from the $\mathrm{CA} 3$ region and septum. $\theta$-frequency and $\theta$-power as well as its synchronization with other neuronal activity both within and outside of the hippocampus (notably the prefrontal cortex) change dynamically with the behavioural state and the cognitive demands faced by the subject.

The function of the $\theta$-rhythm has been the subject of much debate. It is now generally accepted that it plays a role in the mnemonic function of the hippocampus (but $\mathrm{see}^{42}$ for a different view). In most general terms, $\theta$ is thought to serve as an endogenous clock that provides computational windows of synchrony at a frequency of approximately $7-8 \mathrm{~Hz}$ in a rat (baseline $\theta$-frequency varies slightly between species). Within these windows, synchronous multimodal elements of a memory trace, represented by the firing of multiple neuronal assemblies, can be related to each other by their relationship to the phase of the $\theta$-oscillation (Fig. 3). Each $\theta$-cycle (approximately $150 \mathrm{msec}$ in duration) can be envisaged as a temporal envelope holding together multiple bits of correlated information (like a chunk of DNA between start and stop codons). The different bits of information, each a neuronal assembly that forms a coherent representation, are carried by smaller envelopes, the nested $\gamma$-oscillations. These $\gamma$-oscillations, seven of which fit into a $\theta$-cycle, ${ }^{43}$ provide briefer $(\sim 20 \mathrm{msec})$ windows of synchrony. This $\theta$ $\gamma$-nesting (fluctuations of $\gamma$-power as a function of $\theta$-phase) illustrates the intricate interrelationship of neuronal activity on various temporal scales.

A recent computational model, created based on detailed anatomical and physiological studies but also with direct experimental support, ${ }^{33,34,44}$ proposes that the $\theta$-rhythm allows for the rapid switching between conditions that are optimal for memory encoding $v s$ memory retrieval. Strong input from the EC arrives at distal dendrites of CA1 neurons during the trough, whereas strong input from the CA3 region arrives at the peak of the theta wave (peak and trough are referenced to a recording from the hippocampal fissure). Coupled with the $\theta$-phase-dependent propensity for initiating LTP or LTD, effective encoding of new associations occurs optimally when input from EC is strong and LTP of CA3-to-CA1 synapses is most likely, thereby permitting new associations to be encoded effectively without interference from prior learned associations. By contrast, retrieval is optimal when afferent excitation from $\mathrm{EC}$ is weakest, and depotentiation of CA3-to-CA1 synapses during this window allows extinction of prior learned associations that do not match current input. 
The HF offline: consolidating and archiving

When an animal is not actively exploring or planning to explore, the $\theta$-rhythm is replaced by irregular activity of variable amplitude (LIA and SIA) of unclear functional significance. This asynchronous activity is interrupted by SW-Rs, the most synchronous collective events of the hippocampus that are now recognized to play an important role in learning and memory. Firing patterns triggered by recent exploratory experiences (e.g., place cell assemblies firing while the animal runs through a maze) are repeated during SW-R on a compressed time scale, thus reinforcing the connection between them (plasticity) and, as the SW-R leaves the HF, exporting this information from the hippocampus into the cortex..$^{45,46}$

$\theta$-oscillations and sharp waves can be thought of as "companion processes": the former synchronizes the input pathways to the hippocampus, whereas the latter reflects the organized output from the hippocampus back to cortical structures, replaying and transferring information kept in the hippocampus for more permanent storage in the cortex. ${ }^{47,48}$

\section{Anesthetic disruption of hippocampal function: static, dynamic, or both?}

For some drugs, including diazepam and etomidate, there is now good evidence that their ability to induce amnesia arises by way of $\mathrm{GABA}_{\mathrm{A}}$ receptor modulation. In the case of etomidate, which critically depends on $\alpha 5$ subunit modulation, this may well occur primarily within the hippocampus itself. ${ }^{20,49,50}$ Other drugs, such as inhaled anesthetics, may work, at least in part, by this mechanism as well. If so, precisely how does receptor modulation translate to memory impairment?

There are two possible scenarios, which we will term "static" and "dynamic". For the "static" scenario, the ability of repetitive stimuli (such as a $100 \mathrm{~Hz}$ stimulus train) to elicit synaptic plasticity may be suppressed by tilting the balance of synaptic input away from depolarization (excitation) and toward hyperpolarization (inhibition), thus preventing activation of NMDA receptors, calcium entry, and the ensuing cascades that result in LTP. In this regard, the characteristics of $\mathrm{GABA}_{\mathrm{A} \text {,slow }}$ inhibitory postsynaptic currents (IPSCs) are well-suited for this role. Like excitatory synapses, they are localized to the dendrites, and like NMDA receptors, they exhibit a long-lasting conductance. Alternatively, tonic inhibitory influences, which resist depolarization and are sensitive to low drug concentrations, and fast somatic inhibitory synapses, which prevent action potential generation and backpropagation, may also contribute. Perhaps the different mechanisms come into play under different protocols or learning paradigms.
The "dynamic" scenario invokes a more indirect anesthetic action. Since the orchestration of activity by endogenous rhythms in the hippocampus is thought to be essential to its ability to learn and recall activity patterns, might anesthetic-induced amnesia arise from a disruption of some critical aspect of timing required for proper mnemonic function? The type of plasticity an incoming signal elicits in the hippocampus depends on the phase of the $\theta$-oscillation that the signal encounters. ${ }^{51,52}$ Hence, a change in $\theta$-frequency without adjustment of the incoming signalling might interfere with plastic changes underlying memory formation - either preventing its initiation or rendering it too short-lived to support long-term memory storage. Hippocampal interactions with the prefrontal cortex, another important memory-related structure, are also coordinated by the $\theta$-rhythm, ${ }^{53,54}$ with actual information transfer possibly carried by SW-R that require synchronization with cortical rhythms to lay down or reinforce memory traces. ${ }^{46}$ Disruption of this fine-tuned cross-frequency dialogue between the hippocampus and other parts of the brain involved in memory formation, consolidation, and storage degrades mnemonic function. ${ }^{55,56}$ The little evidence that exists to date indicates that anesthetics do not completely eliminate oscillations. However, subtle forms of interference, for example, through changes in $\theta$-frequency, ${ }^{57} \theta$-power, $\theta$ - $\gamma$-nesting, SW-R etc., may all contribute to amnesia via anesthetic actions on a variety of molecular targets.

\section{Conclusions}

Anesthetics' profound interference with behaviour, as witnessed every day in thousands of operating rooms worldwide, is by and large taken as a given instead of the awe-inspiring phenomenon that it truly represents. We and others in the research community perceive that the recognition that anesthesia is not a unitary behavioural state, paired with the capacity of anesthetics to dissociate memory from consciousness, offer an opportunity to gain insight into the workings of the human brain. We hope that this brief primer on the "memory machine" will stimulate the interest of some readers in the mechanisms of anesthetic action.

\section{Glossary}

CA1 to CA3: subdivisions of the hippocampus.

Dentate gyrus: part of the hippocampal formation. Granule cells in the dentate gyrus are the first elements of the excitatory trisynaptic circuit. 
Entorhinal cortex: high-order associational cortex that provides the primary entry port of highly processed information from the cortex to the hippocampus.

$G A B A: \gamma$-aminobutyric acid, principal inhibitory neurotransmitter in the mammalian central nervous system. It activates both ionotropic $\left(\mathrm{GABA}_{\mathrm{A}}\right)$ and metabotropic $\left(\mathrm{GABA}_{\mathrm{B}}\right)$ receptors.

Glutamate: principal excitatory neurotransmitter in the mammalian central nervous system. It activates both ionotropic (AMPA and NMDA) and metabotropic (mGluR) receptors.

Interneurons: also termed local circuit neurons. The prototype interneuron is inhibitory (e.g., GABA-ergic) and does not project outside its brain area (but important exceptions exist).

Ionotropic: receptor/channel that becomes permeable to ions when activated.

Metabotropic: receptor that activates a cascade of 2 nd messengers when activated.

NMDA receptors: subtype of glutamate-activated receptors for which $\mathrm{N}$-Methyl-D-Aspartate is a selective (synthetic) agonist.

Pyramidal cell: The principal cells of the hippocampus and neocortex. Pyramidal cells project to other brain regions using the neurotransmitter glutamate.

Funding Supported by NIH grants GM55719 (MP, RAP) and NS056411 (RAP) and by the Department of Anesthesiology, University of Wisconsin-Madison and the Ralph M. Waters MD endowed professorship (RAP).

Competing interests None declared.

\section{References}

1. Veselis RA, Reinsel RA, Feshchenko VA, Johnson R Jr. Information loss over time defines the memory defect of propofol: a comparative response with thiopental and dexmedetomidine. Anesthesiology 2004; 101: 831-41.

2. Pryor KO, Reinsel RA, Mehta M, Li Y, Wixted JT, Veselis RA. Visual P2-N2 complex and arousal at the time of encoding predict the time domain characteristics of amnesia for multiple intravenous anesthetic drugs in humans. Anesthesiology 2010; 113: 313-26.

3. Brodal A. The hippocampus and the sense of smell; a review. Brain 1947; 70: 179-222.

4. Maclean $P D$. The limbic system with respect to self-preservation and the preservation of the species. J Nerv Ment Dis 1958; 127 : $1-11$.

5. Scoville WB, Milner B. Loss of recent memory after bilateral hippocampal lesions. 1957. J Neuropsychiatry Clin Neurosci 2000; 12: 103-13.

6. Wiltgen BJ, Sanders MJ, Anagnostaras SG, Sage JR, Fanselow $M S$. Context fear learning in the absence of the hippocampus. J Neurosci 2006; 26: 5484-91.
7. Knowlton BJ, Mangels JA, Squire LR. A neostriatal habit learning system in humans. Science 1996; 273: 1399-402.

8. Bechara A, Tranel D, Damasio H, Adolphs $R$, Rockland $C$, Damasio AR. Double dissociation of conditioning and declarative knowledge relative to the amygdala and hippocampus in humans. Science 1995; 269: 1115-8.

9. Eichenbaum $H$. A cortical-hippocampal system for declarative memory. Nat Rev Neurosci 2000; 1: 41-50.

10. Squire LR, Knowlton B, Musen $G$. The structure and organization of memory. Annu Rev Psychol 1993; 44: 453-95.

11. Fanselow MS. Contextual fear, gestalt memories, and the hippocampus. Behav Brain Res 2000; 110: 73-81.

12. Rudy JW, Barrientos RM, O'Reilly RC. Hippocampal formation supports conditioning to memory of a context. Behav Neurosci 2002; 116: 530-8.

13. Eichenbaum $H$. Hippocampus: cognitive processes and neural representations that underlie declarative memory. Neuron 2004; 44: 109-20.

14. Dutton RC, Maurer AJ, Sonner JM, Fanselow MS, Laster MJ, Eger EI 2nd. The concentration of isoflurane required to suppress learning depends on the type of learning. Anesthesiology 2001; 94: 514-9.

15. Dutton RC, Maurer AJ, Sonner JM, Fanselow MS, Laster MJ, Eger EI 2nd. Short-term memory resists the depressant effect of the nonimmobilizer 1-2-dichlorohexafluorocyclobutane (2N) more than long-term memory. Anesth Analg 2002; 94: 631-9.

16. Dutton RC, Maurer AJ, Sonner JM, Fanselow MS, Laster MJ, Eger EI 2nd. Isoflurane causes anterograde but not retrograde amnesia for pavlovian fear conditioning. Anesthesiology 2002; 96: 1223-9.

17. Ghoneim MM, El-Zahaby HM, Block RI. Classical conditioning during nitrous oxide treatment: influence of varying the interstimulus interval. Pharmacol Biochem Behav 1999; 62: 449-55.

18. Alkire MT, Gorski LA. Relative amnesic potency of five inhalational anesthetics follows the Meyer-Overton rule. Anesthesiology 2004; 101: 417-29.

19. Rudolph $U$, Crestani $F$, Benke D, et al. Benzodiazepine actions mediated by specific gamma-aminobutyric acid(A) receptor subtypes. Nature 1999; 401: 796-800.

20. Cheng VY, Martin LJ, Elliott EM, et al. Alpha5GABAA receptors mediate the amnestic but not sedative-hypnotic effects of the general anesthetic etomidate. J Neurosci 2006; 26: 3713-20.

21. Grunwald T, Beck H, Lehnertz K, et al. Evidence relating human verbal memory to hippocampal N-methyl-D-aspartate receptors. Proc Natl Acad Sci USA 1999; 96: 12085-9.

22. Tsien JZ. Linking Hebb's coincidence-detection to memory formation. Curr Opin Neurobiol 2000; 10: 266-73.

23. Martin SJ, Grimwood PD, Morris RG. Synaptic plasticity and memory: an evaluation of the hypothesis. Annu Rev Neurosci 2000; 23: 649-711.

24. Hemmings HC Jr, Akabas MH, Goldstein PA, Trudell JR, Orser $B A$, Harrison $N L$. Emerging molecular mechanisms of general anesthetic action. Trends Pharmacol Sci 2005; 26: 503-10.

25. Zhang $Y$, Laster MJ, Hara $K$, et al. Glycine receptors mediate part of the immobility produced by inhaled anesthetics. Anesth Analg 2003; 96: 97-101.

26. Heurteaux C, Guy N, Laigle C, et al. TREK-1, a $\mathrm{K}(+)$ channel involved in neuroprotection and general anesthesia. EMBO J 2004; 23: 2684-95.

27. Lazarenko RM, Willcox SC, Shu S, et al. Motoneuronal TASK channels contribute to immobilizing effects of inhalational general anesthetics. J Neurosci 2010; 30: 7691-704.

28. Simon W, Hapfelmeier G, Kochs E, Zieglgansberger W, Rammes $G$. Isoflurane blocks synaptic plasticity in the mouse hippocampus. Anesthesiology 2001; 94: 1058-65. 
29. Pearce RA, Stringer JL, Lothman EW. Effect of volatile anesthetics on synaptic transmission in the rat hippocampus. Anesthesiology 1989; 71: 591-8.

30. Braitenberg V, Schuz A. Some anatomical comments on the hippocampus. In: Seifert W, editor. Neurobiology of the hippocampus. London: Academic Press Inc.; 1983. p. 21-37.

31. Haberly $L B$. Parallel-distributed processing in olfactory cortex: new insights from morphological and physiological analysis of neuronal circuitry. Chem Senses 2001; 26: 551-76.

32. Ylinen A, Bragin A, Nadasdy Z, et al. Sharp wave-associated high-frequency oscillation $(200 \mathrm{~Hz})$ in the intact hippocampus: network and intracellular mechanisms. J Neurosci 1995; 15: 3046.

33. Villarreal DM, Gross AL, Derrick BE. Modulation of CA3 afferent inputs by novelty and theta rhythm. J Neurosci 2007; 27 : 13457-67.

34. Colgin LL, Denninger T, Fyhn M, et al. Frequency of gamma oscillations routes flow of information in the hippocampus. Nature 2009; 462: 353-7.

35. McHugh TJ, Jones MW, Quinn JJ, et al. Dentate gyrus NMDA receptors mediate rapid pattern separation in the hippocampal network. Science 2007; 317: 94-9.

36. Rolls ET, Kesner RP. A computational theory of hippocampal function, and empirical tests of the theory. Prog Neurobiol 2006; 79: $1-48$.

37. Leutgeb JK, Leutgeb S, Moser MB, Moser EI. Pattern separation in the dentate gyrus and CA3 of the hippocampus. Science 2007; 315: 961-6.

38. Lisman JE. Relating hippocampal circuitry to function: recall of memory sequences by reciprocal dentate-CA3 interactions. Neuron 1999; 22: 233-42.

39. Vinogradova OS. Hippocampus as comparator: role of the two input and two output systems of the hippocampus in selection and registration of information. Hippocampus 2001; 11: 578-98.

40. Ang CW, Carlson GC, Coulter DA. Hippocampal CA1 circuitry dynamically gates direct cortical inputs preferentially at theta frequencies. J Neurosci 2005; 25: 9567-80.

41. Leung $L W$. Model of gradual phase shift of theta rhythm in the rat. J Neurophysiol 1984; 52: 1051-65.

42. Bland $B H$, Oddie $S D$. Theta band oscillation and synchrony in the hippocampal formation and associated structures: the case for its role in sensorimotor integration. Behav Brain Res 2001; 127: 119-36.

43. Lisman J, Buzsaki G. A neural coding scheme formed by the combined function of gamma and theta oscillations. Schizophr Bull 2008; 34: 974-80.

44. Manns JR, Zilli EA, Ong KC, Hasselmo ME, Eichenbaum H. Hippocampal CA1 spiking during encoding and retrieval: relation to theta phase. Neurobiol Learn Mem 2007; 87: 9-20.
45. Buzsaki G. The hippocampo-neocortical dialogue. Cereb Cortex 1996; 6: 81-92.

46. Siapas $A G$, Wilson MA. Coordinated interactions between hippocampal ripples and cortical spindles during slow-wave sleep. Neuron 1998; 21: 1123-8.

47. Buzsaki G. Two-stage model of memory trace formation: a role for "noisy" brain states. Neuroscience 1989; 31: 551-70.

48. Chrobak JJ, Buzsaki G. High-frequency oscillations in the output networks of the hippocampal-entorhinal axis of the freely behaving rat. J Neurosci 1996; 16: 3056-66.

49. Belelli D, Lambert JJ, Peters JA, Wafford K, Whiting PJ. The interaction of the general anesthetic etomidate with the gammaaminobutyric acid type A receptor is influenced by a single amino acid. Proc Natl Acad Sci USA 1997; 94: 11031-6.

50. Martin LJ, Zurek AA, MacDonald JF, Roder JC, Jackson MF, Orser BA. Alpha5GABAA receptor activity sets the threshold for long-term potentiation and constrains hippocampus-dependent memory. J Neurosci 2010; 30: 5269-82.

51. Huerta PT, Lisman JE. Bidirectional synaptic plasticity induced by a single burst during cholinergic theta oscillation in CA1 in vitro. Neuron 1995; 15: 1053-63.

52. Hyman JM, Wyble BP, Goyal V, Rossi CA, Hasselmo ME. Stimulation in hippocampal region CA1 in behaving rats yields long-term potentiation when delivered to the peak of theta and long-term depression when delivered to the trough. J Neurosci 2003; 23: 11725-31.

53. Siapas AG, Lubenov EV, Wilson MA. Prefrontal phase locking to hippocampal theta oscillations. Neuron 2005; 46: 141-51.

54. Hyman JM, Zilli EA, Paley AM, Hasselmo ME. Working memory performance correlates with prefrontal-hippocampal theta interactions but not with prefrontal neuron firing rates. Front Integr Neurosci 2010; 4: 2.

55. Girardeau G, Benchenane K, Wiener SI, Buzsaki G, Zugaro MB. Selective suppression of hippocampal ripples impairs spatial memory. Nat Neurosci 2009; 12: 1222-3.

56. Ego-Stengel V, Wilson MA. Disruption of ripple-associated hippocampal activity during rest impairs spatial learning in the rat. Hippocampus 2010; 20: 1-10.

57. Perouansky $M$, Hentschke H, Perkins M, Pearce RA. Amnesic concentrations of the nonimmobilizer 1,2-dichlorohexafluorocyclobutane $(\mathrm{F} 6,2 \mathrm{~N})$ and isoflurane alter hippocampal theta oscillations in vivo. Anesthesiology 2007; 106: 1168-76.

58. Jensen $O$, Lisman JE. An oscillatory short-term memory buffer model can account for data on the Sternberg task. J Neurosci 1998; 18: 10688-99. 